\title{
A HIGH EFFICIENCY INTERLEAVED PFC FRONT - END CONVERTER FOR EV BATTERY CHARGER
}

Nowadays, an increasingly present application in the automotive field is the battery charger that usually consists of two high efficiency parts: an AC/DC converter with power factor correction (PFC) capability and a DC/DC converter. In this paper, a three-channel interleaved Power Factor Correction (PFC) based on a new digital controller STNRGPF01 operating in Continuous Conduction Mode (CCM) is presented addressing high efficiency. In the considered applications, the most challenging control issues are the input current control and the fast overcurrent protections. Exploiting mixed signal control approach some benefits have been obtained and confirmed by experimental results on a $3 \mathrm{~kW}$ prototype.

Keywords: active front-end, interleaved converters, battery charger, power factor correction, digital control

\section{Introduction}

For electric vehicle manufacturers (EVs) and for the consumer market it is important to have high efficiency and high power chargers to optimize charging times. The charger consists of an $\mathrm{AC} / \mathrm{DC}$ used as power factor correction (PFC), and a DC/ $\mathrm{DC}$ to adapt the voltage levels to the battery type. If the efficiency of each part is greater than $97 \%$, the overall efficiency of the battery charger will be $94 \%$ higher, unlike most of the chargers on the market [1].

In many high power applications active Power Factor Corrector (PFC) converters are widely used as the first stage in $\mathrm{AC} / \mathrm{DC}$ conversion in order to meet the IEC 61000-3-2 standard for electrical equipment [2].

In order to charge the battery pack of Electrical Vehicles (EVs) from the grid, a battery charger with unity power factor correction (PFC) capacity is required. For the on-board charger (OBC) with two-stage structure [3], the front-end PFC AC/DC converter is used to rectify the input supply from $\mathrm{AC}$ form to DC form while controlling the input current of converter to be in phase with the input voltage, therefore, high power factor control is achieved to minimize the negative effect of $\mathrm{OBC}$ on the power quality of the grid. The result consists in an electrical appliance that work virtually as a pure resistive load thus the overall grid efficiency can be improved.
The conventional boost topology is the most popular topology used to realize PFC and ac/dc conversion in the power range of a few kilowatts. Generally, the converter is controlled based on the conventional dual closed-loop PI control, among which an external voltage loop regulates the output voltage and generates the reference current and an internal current loop shapes the input current to achieve the unity power factor correction [4].

In applications of power greater than $1 \mathrm{~kW}$, interleaved converters are often used. Interleaving of PFCs consists of paralleling two or more active stages (Figure 1), each rated for a fractional power, instead of a bigger one rated for the full power. During normal operation the PWM driving signals are out of phase of a proper value according to sequent equation:

phase shift $=\frac{360^{\circ}}{\text { number of channels }}$

The benefits of the interleaved topology [5], compared to traditional single-stage PFC, are measured in terms of:

- Input current ripple reduction

- $\quad$ EMI filter volume reduction

- $\quad$ Inductor volume reduction

- $\quad$ Output capacitor RMS current value reduction

- $\quad$ Better power management for the switches

- $\quad$ higher efficiency thanks to channels power management

\footnotetext{
* ${ }^{1}$ Giuseppe Aiello, ${ }^{1}$ Mario Cacciato, ${ }^{2}$ Sebastiano Messina, ${ }^{2}$ Marco Torrisi

${ }^{1}$ Department of Electrical, Electronics Engineering and Computer Science, University of Catania, Italy

${ }^{2}$ STMicroelectronics, Catania, Italy

E-mail: mario.cacciato@unict.it
} 


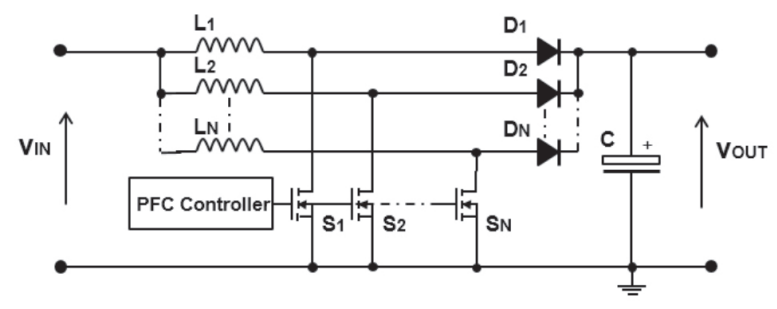

Figure 1 Interleaved boost PFC with $N$ active channels

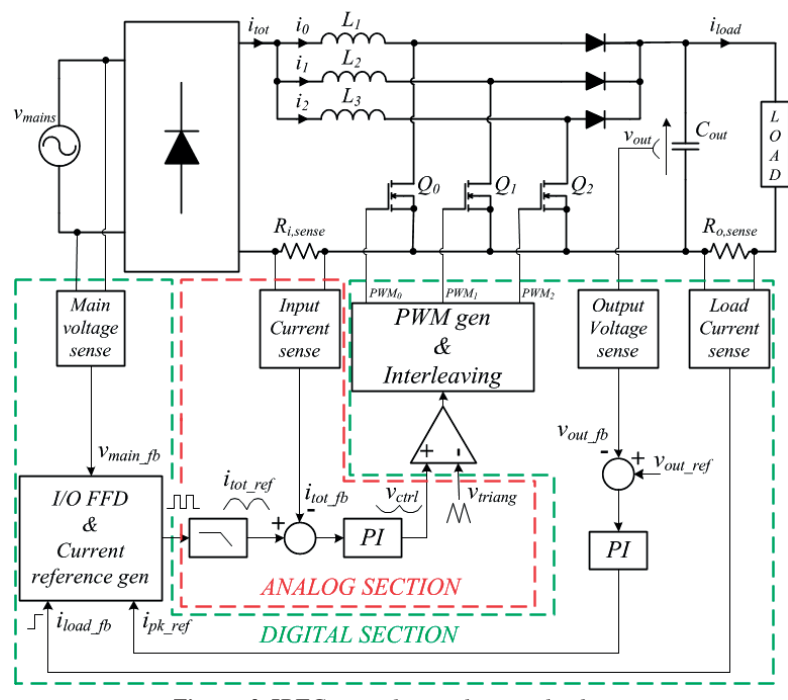

Figure 2 IPFC mixed signal control scheme

\section{Mixed signal concept}

If only Analog ICs are used, generally it not allows to totally fulfill the multi specification of power converter. For such a reason, full digital solutions are often preferred to analog ones for their flexibility and programmability. Consequently, costly microcontrollers with high computational power are needed to meet the high bandwidth requested by the current control loops. Moreover, full digital control has bandwidth limitations compared to analog implementation [6 - 8].

As a consequence, the mixed-signal control represents a good compromise because it provides at the same time the flexibility of digital solution and the dynamics of analog controllers. For the addressed application, the proposed control scheme is shown in Figure 2 [9].

An outer voltage control loop is used to set the PFC bus voltage to a reference value while an inner current loop regulates the value of the total average inductor current. A digital PI regulator calculates the peak of total input average current ipk_ref by considering the difference between the output voltage feedback Vout_fb and the reference Vout_ref. In this scheme, the PFC current reference is obtained from the I/O Feed Forward (I/O FFD) block exploiting a PWM waveform filtered using a low pass analog filter to achieve the pre-modulation reference waveform. Then, the difference between the current reference itot_ref and

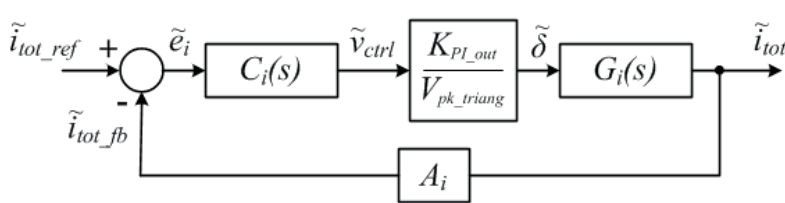

Figure 3 PFC current control loop block diagram

the input current feedback itot_fb is processed by an analog PI controller whose output vctrl is compared with a triangular wave Vtriang at the switching frequency to generate the master signal PWM0. In Figure 2, the digital and analog sections are highlighted by the green and red dashed line respectively. Input and load feed-forward control are used to ensure fast dynamic response when main voltage changes suddenly or a load current step occurs. Moreover, in order to obtain an almost flat figure of merit a phase shedding function is implemented in order to enable/disable the slave channels according to the load.

\section{Theoretical analysis of control loops}

\subsection{Current control loop design}

The small-signal transfer functions of the interleaved boost converter are obtained using the State-Space Averaging (SSA) method and a linearization operation (Taylor's series around an operating point) $[10,11]$. In order to simplify the problem, the following assumption has been considered:

- Converter CCM operation

- Ideal active and passive components

- The three boost inductors show identical parameters and the total power is equally split among the channels

- The grid voltage is assumed to be stable during several switching cycles.

In the following equations the notation " , specifies smallsignal variables while the uppercase letter indicates quantities operating in steady-state. The control-to-input current transfer function is shown in Equation 2.

$G_{i}(s)=\frac{\tilde{i}_{\text {tot }}}{\tilde{\delta}}=\frac{C_{\text {OUT }} V_{\text {OUT }}^{3} s+P_{\text {OUT }}\left(1+\frac{1}{\eta}\right) V_{\text {OUT }}}{C_{\text {OUT }} L_{P F C} V_{\text {OUT }}^{2}+L_{P F C} P_{\text {OUT }} s+N_{c h} V_{I N}^{2}}$

The current control loop block diagram is shown in Figure 3.

Where Vpk_triang is the peak to peak voltage of the triangular carrier and KPI_out is a scaling factor that allow to match the PI regulator maximum output voltage value with that of the triangular carrier peak.

$\mathrm{Ai}$ is the input current sensing gain and $\mathrm{Ci}(\mathrm{s})$ is the compensator transfer function. The open loop compensated transfer function is then given by:

$T_{i}(s)=C_{i}(s) \frac{K_{P_{\text {oou }}}}{V_{p k_{-} \text {tirang }}} A_{i} G_{i}(s)$ 


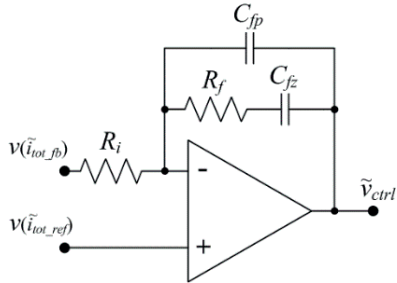

Figure 4 Type II compensation amplifier

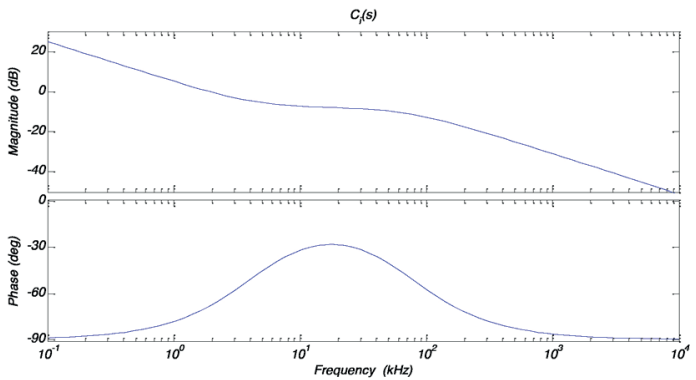

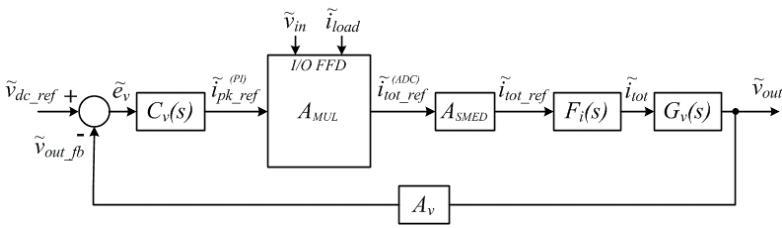

Figure 5 Complete cascaded control loop block diagram

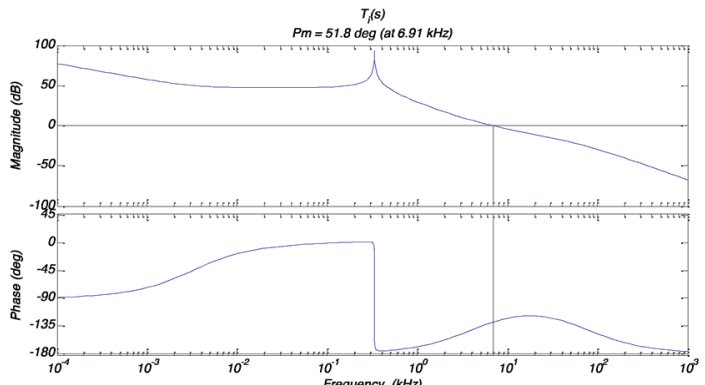

Figure 6 Bode diagrams of current compensator $C_{i}(s)$ and current open loop $T_{i}(s)$ transfer functions

where the typical PI controller transfer function is $C_{i}(s)=\left(K_{P_{-} \text {Itot }} s+K_{I_{\text {Ittot }}}\right) / \mathrm{s}$. Based on general Bode criteria equation - Equation (4) ensure system stability for a desired bandwidth $\omega_{T i \text { des }}$ (crossover pulsation) and phase margin $P M_{i \text { des }}$ and the PI controller parameters can be calculated from Equation (5)

$$
\begin{aligned}
& \int\left|T_{i}\left(j \omega_{\text {Ti_des }}\right)\right|=1 \\
& \left\{\angle T_{i}\left(j \omega_{T i \_d e s}\right)=-180^{\circ}+P M_{i \_ \text {des }}\right.
\end{aligned}
$$

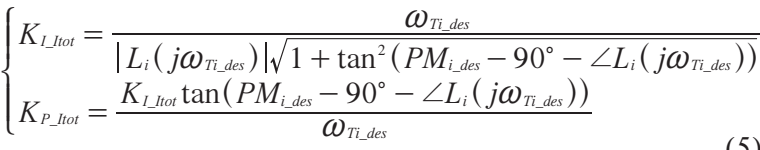

Typically, the crossover frequency is selected within the range $2-10 \mathrm{kHz}$ [12], while phase margin $45-60$ degrees.

Since the current controller is implemented with analog type - II compensation amplifier (Figure 4) the transfer function of this compensator is given by Equation (5).

$$
C_{i}(s)=\frac{1}{\left(C_{f z}+C_{f p}\right) R_{i}} \frac{C_{f z} R_{f} s+1}{s\left(\frac{C_{f z} C_{f p} R_{f}}{C_{f z}+C_{f p}} s+1\right)}
$$

\subsection{Voltage loop design}

The input current-to-output voltage transfer function is specified in Equation (7):

$$
G_{v}(s)=\frac{\tilde{\nu}_{\text {out }}}{\tilde{i}_{\text {tot }}}=\frac{2\left(N_{\text {ch }} V_{I N}-\frac{P_{\text {OUT }}}{\eta V_{I N}} L_{P F C} S\right) V_{\text {OUT }}^{2}}{C_{\text {OUT }} V_{\text {OUT }}^{3} S+P_{\text {OUT }}\left(1+\frac{1}{\eta}\right) V_{\text {OUT }}}
$$

voltage loop crossover frequency is generally selected in the range $5-15 \mathrm{~Hz}$ [12], thus the right half plane zero (higher frequency) can be neglected. The cascaded control block diagram is shown in Figure 5.

where:

$F_{i}(\mathrm{~s})=$ Input current closed-loop transfer function

$C_{v}(\mathrm{~s})=$ Output voltage compensator transfer function

$A_{M U L}=$ Digital multiplier gain for digital current reference generation

$A_{S M E D}=$ Digital to analog gain for analog current reference generation

$A_{v}=$ Output voltage sensing gain

The I/O FFD block can be simply considered as a constant gain block $A_{M U L}$. The output voltage loop regulation is done with a digital PI controller:

$C_{v}(s)=\frac{K_{P_{-} V d c} S+K_{I_{-} V d c}}{s}$

Considering the open loop compensated transfer function shown in Equation (9):

$$
T_{v}(s)=C_{v}(s) A_{M U L} A_{\text {SMED }} F_{i} G_{v}(s) A_{v}
$$

and that stable system is obtained if the two following conditions are verified:

$\left\{\begin{array}{l}\left|T_{v}\left(j \omega_{T v_{\text {des }}}\right)\right|=1 \\ \angle T_{v}\left(j \omega_{T v_{\text {des }}}\right)=-180^{\circ}+P M_{v_{v} \text { des }}\end{array}\right.$ 

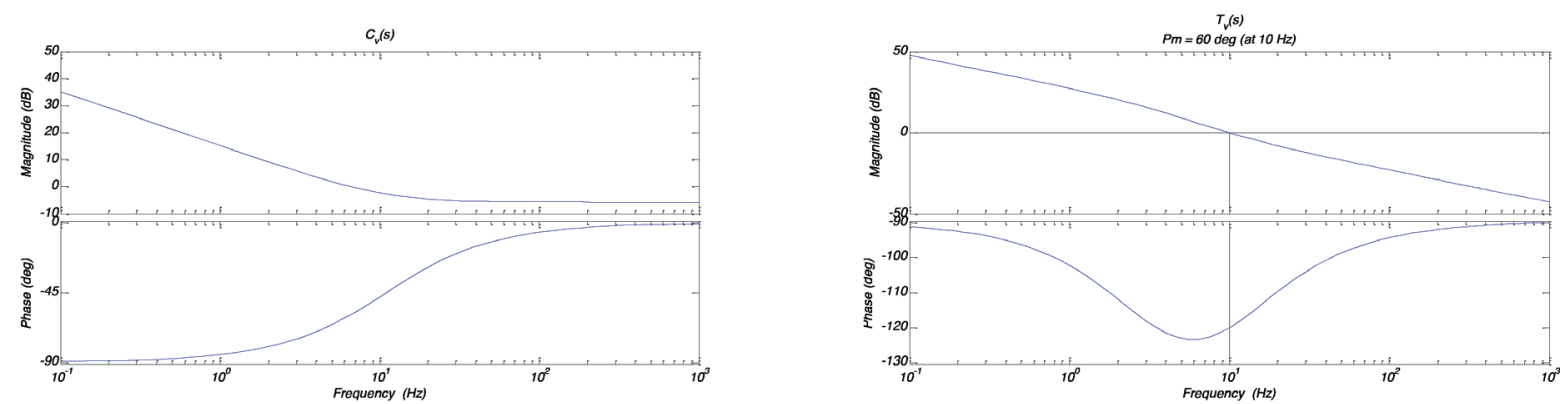

Figure 7 Bode diagrams of voltage compensator $C_{v}(s)$ and voltage open loop $T_{v}(s)$ transfer functions

Table $13 \mathrm{~kW}$ three-channel IPFC prototype specifications

\begin{tabular}{|c|c|c|}
\hline Design parameter & Description & Value \\
\hline$P_{\text {OUT }}$ & output power & 3 kW@230 Vac \\
\hline$N_{c h}$ & Number of channels & 3 \\
\hline$V_{I N}$ & rms nominal input voltage & $230 \mathrm{~V}$ \\
\hline$V_{\text {oUT }}$ & rms nominal output voltage & $400 \mathrm{~V}$ \\
\hline$f$ & line frequency & $50-60 \mathrm{~Hz}$ \\
\hline$\eta$ & estimated efficiency & $98 \%$ \\
\hline$L_{P F C}$ & single channel boost inductor & $120 \mu \mathrm{H}$ \\
\hline$C_{\text {OUT }}$ & output capacitor & $4 \mathrm{x} 470 \mu \mathrm{F}$ \\
\hline$V_{p k \_t r i a n g}$ & peak-to-peak voltage of triangular wave & $2 \mathrm{~V}$ \\
\hline$K_{\text {PI_out }}$ & PI out scale factor & 0.5909 \\
\hline$A_{i}$ & Input current sensing gain & 0.0927 \\
\hline$A_{v}$ & Output voltage sensing gain & 1.9128 \\
\hline$A_{M U L}$ & Digital multiplier gain & 3.3086 \\
\hline$A_{S M E D}$ & Digital to analog gain & 0.00068 \\
\hline$f_{s w}$ & Switching frequency & $111 \mathrm{kHz}$ \\
\hline$f_{T i \_d e s}$ & Current loop crossover frequency & $7.5 \mathrm{kHz}$ \\
\hline$f_{T v_{-} \text {des }}$ & Voltage loop crossover frequency & $10 \mathrm{~Hz}$ \\
\hline$P M_{i \_d e s}$ & Current loop phase margin & $60^{\circ}$ \\
\hline$P M_{v \text { des }}$ & Voltage loop phase margin & $60^{\circ}$ \\
\hline
\end{tabular}

The compensator parameters can be obtained by solving the following system:

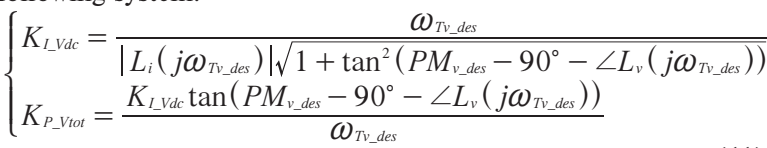

Finally, according to Table 1 specifications, Figure 6 and Figure 7 show respectively the Bode diagrams for current and voltage loops. Because of the high-frequency pole and standard passive components values the actual phase margin and crossover frequency of $\mathrm{Ci}(\mathrm{s})$ are lower than the target values $\left(60^{\circ}, 7.5 \mathrm{kHz}\right)$, therefore it has to be compensated by imposing a slightly larger value as specific.

\section{Experimental results}

A $3 \mathrm{~kW}$ three-channel IPFC prototype, showed in Figure 8 , has been designed and realized in order to test the proposed control scheme, according to the following specifications - see Table 1.

The power density of $52 \mathrm{~W} /$ inch $^{3}$ is achieved exploiting a compact layout and the small size of magnetic components related with the benefits of interleaving and high switching frequency.

Figure 9 shows the inductor currents waveforms in small time scale. The interleaving operation is properly set with 120 degrees of phase shift. 


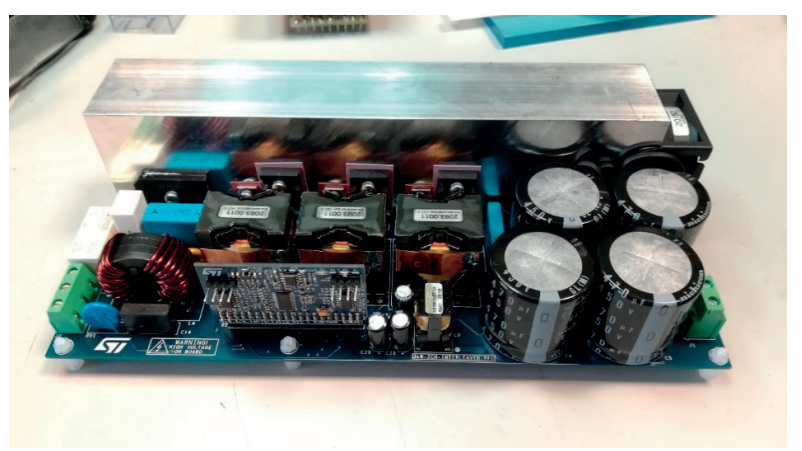

Figure $83 \mathrm{~kW}$ 3-channel interleaved PFC prototype

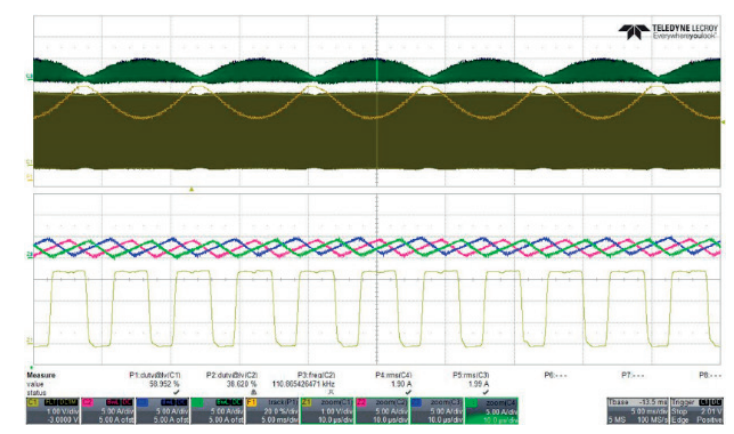

Figure 9 Inductor current waveforms at switching frequency

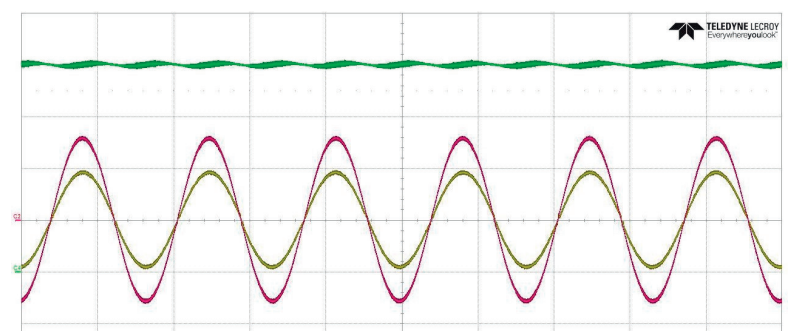

Input current (C1) Input voltage (C2) Output voltage (C4)

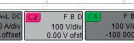

Figure $10 \mathrm{IPFC}$ at $1.5 \mathrm{~kW}(115 \mathrm{Vac}, 60 \mathrm{~Hz})$

The waveform in steady state at line frequency are shown in Figure 10 and Figure 11. The board is supplied by a power AC source at $115 \mathrm{Vac}$ and the power is de-rated for thermal reasons down to $1.5 \mathrm{~kW}$ while it is $3 \mathrm{~kW}$ when connected to the $230 \mathrm{Vac}$ grid. The input current faithfully follows the reference thus an almost sinusoidal shaped waveform with near unity power factor is obtained even in case of line voltage distortion.

The control fast dynamic response is clearly stated in Figure 12 where a load step from $10 \%$ to $100 \%$ and again to $10 \%$ is considered. Thanks to the load feed-forward the output voltage fluctuations during load transitions have been reduced.

In steady state condition THD, PF and efficiency performance are evaluated. Figure 13 shows a THD lower than $10 \%$ at loads higher than $20 \%$. It decreases down to $3 \%$ at full load for $230 \mathrm{Vac}$

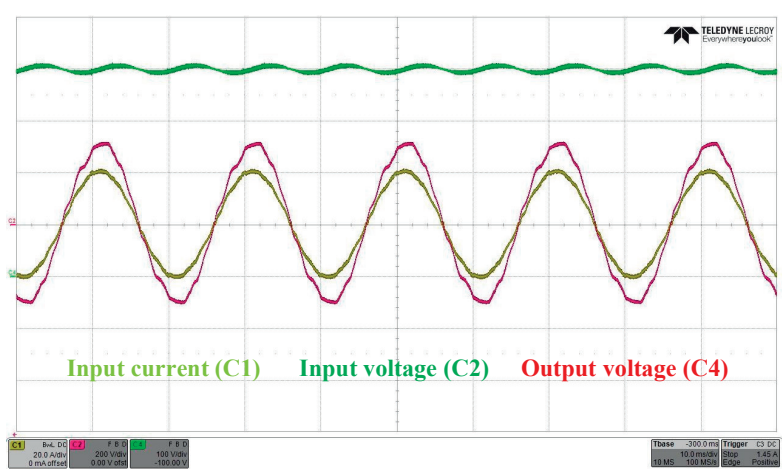

Figure $11 \mathrm{IPFC}$ at $3 \mathrm{~kW}(230 \mathrm{Vac}, 50 \mathrm{~Hz})$

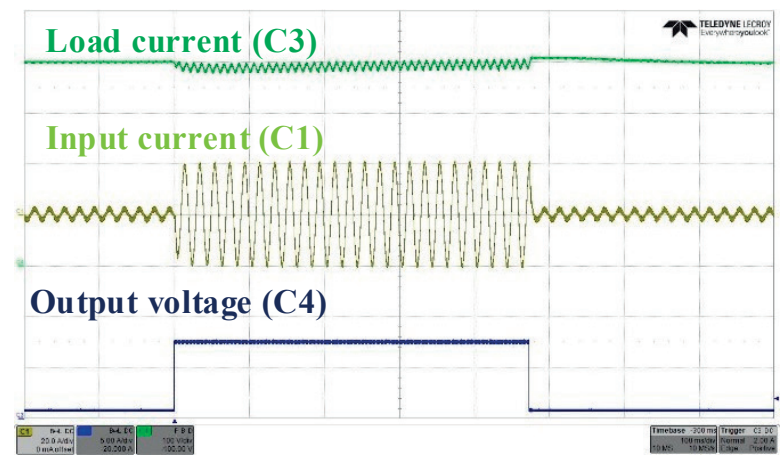

Figure 12 10\% - 100\% - 10\% load transition

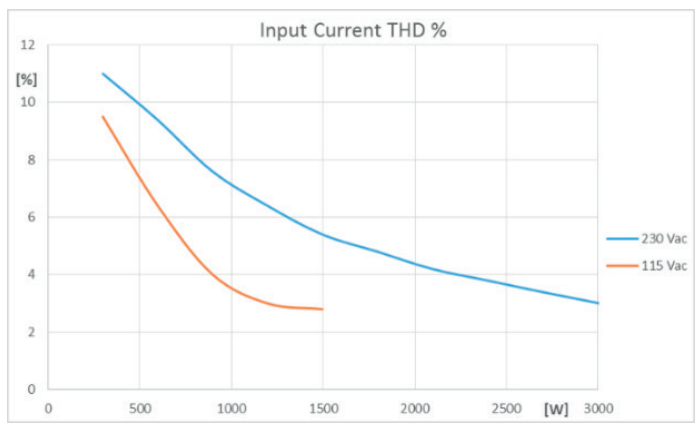

Figure 13 Input Current THD \%

and below $3 \%$ for 115 Vac. High power factor (> 0.99$)$ is achieved from $20 \%$ load.

Finally, a high and nearly flat efficiency has been obtained and showed in Figure 14 thanks to the phase shedding operation. Its value is about $97.6 \%$ from $50 \%$ to $100 \%$ load.

\section{Conclusion}

For automotive application of PFC AC/DC converter to be used as front-end stage in battery charger, a new approach is presented combining mixed signal control and phases interleaving that allows to match at the same time the advantages of analog 

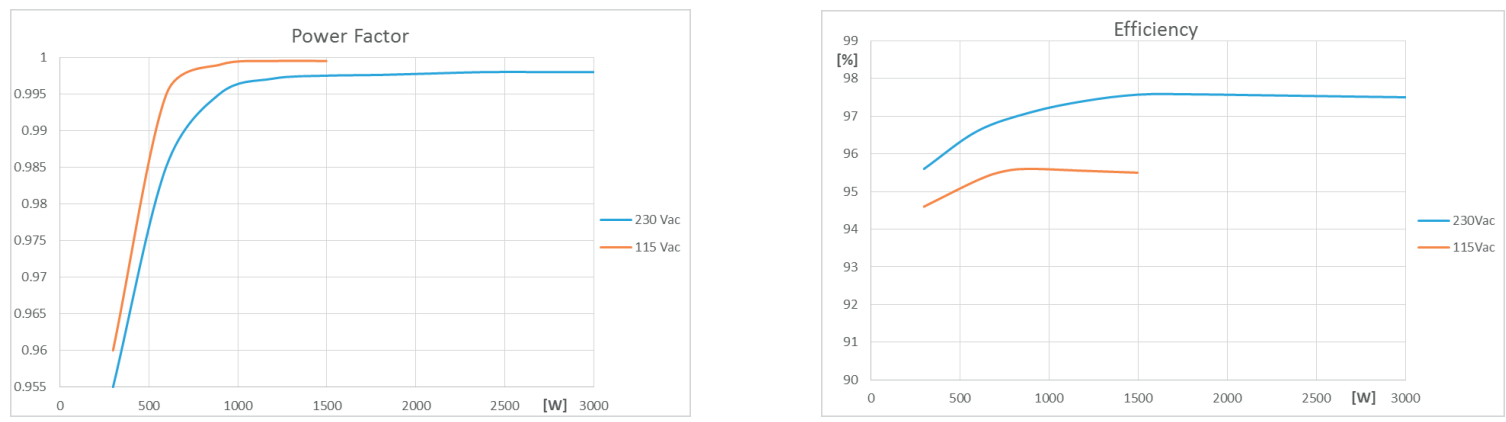

Figure 14 Power factor vs output PFC power (left) and converter efficiency (right)

cycle by cycle regulation and the flexibility of a digital system still performing high effiency.

In the paper, theoretical aspects have been considered, the design of both the analog and the digital control loops is introduced and the procedure for the control implementation has been provided.
A prototype has been realized in order to test the proposed control scheme. THD of $3 \%$ and PF well above 0.99 are achieved for the rated power, the measured efficiency at $230 \mathrm{Vac}$ is just below $98 \%$ and shows a nearly flat behavior. Moreover, input current waveform is not affected from grid voltage distortion and test results show very good performances for both steady-state and dynamic conditions.

\section{References}

[1] Develops Prototype Medium-Voltage Utility Direct Fast Charger. Epri Journal, Spring, 2012.

[2] CONSOli, A., CACCIATO, M., TESTA, A., GENNARO, F.: Single Chip Integration for Motor Drive Converters with Power Factor Capability. IEEE Transactions on Power Electronics, 19(6), 1372-1379, 2004.

[3] YOUNG-JOO LEE, KHALIGH, A., EMADI, A.: Advanced Integrated Bidirectional AC/DC and DC/DC Converter for Plug-In Hybrid Electric Vehicles. IEEE Transactions on Vehicular Technology, 58(8), 3970-3980, 2009.

[4] LU, Y. W., ZHANG, W., LIU, Y.: A Large Signal Dynamic Model for Single Phase AC-To-DC Converters with Power Factor Correction. Proceedings of 35th Annual IEEE Power Electronics Specialists Conference, Germany, 2, 1057-1063, 2004.

[5] Power Factor Correction (PFC) Handbook: Choosing the Right Power Factor Controller Solution [online], 2014, SCILLC, USA, rev. 5, 2014

[6] ZHANG, W., FENG, G., LIU, Y., WU, B.: A Digital Power Factor Correction (PFC) Control Strategy Optimized for DSP. IEEE Transactions on Power Electronics, 19(6), 1474-1485, 2004.

[7] CACCiATO, M., SCARCElla, G., SCElBA, G., FINOCCHIARO, L.: Multi-Reference Frame Based PLL for Single Phase Systems in Voltage Distorted Grids. Proceedings of 16th European Conference on Power Electronics and Applications, Finland, $1-8,2014$.

[8] CACCIATO, M., CONSOli, A., AiEllo, N., ATTANASIO, R., GENNARO, F., MACINA, G.: A Digitally Controlled Double Stage Soft-Switching Converter for Grid-Connected Photovoltaic Applications. Proceedings of 23rd Annual IEEE Applied Power Electronics Conference and Exposition, USA, 141-147, 2008.

[9] STNRGPF01 Three-channel interleaved CCM PFC digital controller, Data Sheet DS10246 [online]. STMicroelectronics, 2017.

[10] NAGULAPATI, K., RANGAVALLI, V., VANAJAKSHI, B.: Modeling, Analysis and Simulation of 4-Phase Boost Converter. International Journal of Electrical, Computer, Electronics and Communication Engineering, 8(9), 1452-1462, 2014.

[11] BRYANT, B., KAZIMIERCZUK, M. K.: Open-Loop Power-Stage Transfer Functions Relevant to Current-Mode Control of Boost PWM Converter Operating in CCM. IEEE Transactions on Circuits and Systems-I: regular papers, 52(10), 2158-2164, 2005.

[12] MORICONI, U.: Designing a High Power Factor Switching Pre-Regulator with the L4981 Continuous Mode, Application Note AN628 [online]. STMicroelectronics, 2004. 